\title{
Control of disability glare by means of electrochromic filtering glasses: A pilot study
}

\author{
Rosa Maria Coco-Martín*,\|, Rubén Cuadrado-Asensio*, César Vega-Colado ${ }^{\dagger}$, \\ Ricardo Vergaz ${ }^{\dagger}$, José M. Sánchez-Pena ${ }^{\dagger}$, Ana Viñuales ${ }^{\star}$, Sergi Peris ${ }^{\S}$, \\ Pau Artús ${ }^{\S}$ and M. Begoña Coco-Martín*, \\ *IOBA, Universidad de Valladolid, Valladolid, Spain \\ ${ }^{\dagger}$ GDAF-UC3M, Grupo de Displays y Aplicaciones Fotónicas \\ Department de Tecnología Electrónica \\ Universidad Carlos III de Madrid, Spain \\ \$IK4-CIDETEC, Parque Tecnológico de San Sebastián \\ Guipuzcoa, Spain

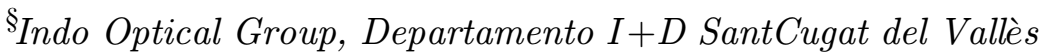 \\ Barcelona, Spain \\ "GIDFYS, European University Miguel de Cervantes(UEMC) \\ Department of Health, Sciences, Spain \\ "rosa@ioba.med.uva.es
}

Received 19 October 2015

Accepted 29 December 2015

Published 4 March 2016

\begin{abstract}
Purpose: To evaluate the potential clinical usability of a new prototype of ophthalmic blue light filters developed by using electrochromic technology in pseudophakic patients complaining of glare. Methods: A prototype of electrochromic device was developed, with a specific frame that enclosed an electronic driver that allowed personalizing its function for each patient. A prospective, observational case series study was performed to test it. Five patients who had undergone cataract surgery with clear intraocular lenses and complaining of glare were included in the study. Main outcome measures were the results obtained in the satisfaction questionnaire that was delivered to patients. Then, visual acuity, contrast sensitivity, and reading ability data were evaluated with and without the prototype under different lighting conditions and different modes of the prototype after a complete month of use. Lens transmittance was also measured. Results: Visual acuity, contrast sensitivity and reading function did not change significantly with prototype use. The main activity for which the prototype was used was walking. Only one patient found that the dimming level was insufficient. No patients reported variable discomfort when passing tunnels, not sufficiently clear indoors, or put on and remove discomfort. The lenses slightly decreased their transmittance at the end of the study. Conclusion: Glasses based on
\end{abstract}

\|Corresponding author.

This is an Open Access article published by World Scientific Publishing Company. It is distributed under the terms of the Creative Commons Attribution 4.0 (CC-BY) License. Further distribution of this work is permitted, provided the original work is properly cited. 
electrochromic technology may be acceptable for outdoor/indoor use and for distance-near vision. Future studies with larger samples must be conducted to confirm the clinical usability of these glasses.

Keywords: Optical filters; electro-optical devices; technical visual aids; electronic control.

\section{Introduction}

Cataract surgery is one of the most frequent procedures performed in the world and its rate has been rising over the last years. ${ }^{1}$ Following cataract surgery, a discrepancy is often encountered between the visual function measured by visual acuity (VA) and the visual disability experiences. Patients can present a variety of visual complaints such as haloes, ghost images or glare, despite having good VA. ${ }^{2}$ Glare results from an increase in intraocular forward light scattering, which has also been proposed as the primary cause for reduced retinal contrast and veiling luminance on the retina. ${ }^{3}$ Spectral filters, which absorb short wavelength light, have been suggested to provide some visual benefits in pseudophakic individuals. ${ }^{4}$ However, patients could need a different filter for each lighting environment, ${ }^{5,6}$ forcing them to carry several glasses.

Currently, filtering lenses on the market are passive (it does not matter if their coloration is constant or active) when the color and transmittance change according to the surrounding conditions. These active filtering lenses are commonly photochromic lenses which have some limitations: ultraviolet (UV) radiation is needed to activate them, change takes several minutes, and they are strongly temperature dependent. ${ }^{7}$ Current active materials can control light transmission, reflection, scattering, or absorption processes by using an external electrical voltage or a current signal. ${ }^{8}$ As these materials can modulate their color, they have been coined with the term chromogenic. ${ }^{9}$ Likewise, these material can be subdivided into three main types: electrophoretic materials, liquid crystals, and electrochromic materials. Electrophoretic materials are not used in visual applications as they usually show very low or no light transmittance. Liquid crystals act reorienting their molecules, changing the effective refractive index or the polarization angle of light, whereas electrochromic materials (ECs) use a redox reaction to change their light absorption properties. Some devices have been developed using this type of active materials, such as sunglasses ${ }^{10}$ or magnifying glasses, although attempts to use ECs in sunglasses have not been successful to this date. ${ }^{11-13}$

This paper describes an EC device designed and prototyped expressly for applying in spectacles as ophthalmic active filters. The proof of concept was implemented in a frame designed and programmed in a microcontroller. The manufactured glasses were tested in pseudophakic patients, each receiving his/ her customized glasses with a specific pair of EC lenses. The aim of this study was to evaluate the influence of the EC filters on the visual function and patient satisfaction.

\section{Material and Methods}

A prospective, observational case series study was performed. Data gathering was carried out after the local ethics committee approved the study protocol and informed consent was obtained from each patient. This research followed the tenets of the Declaration of Helsinki.

\subsection{Electrochromic glasses}

\subsubsection{Electrochromic material}

A highly transparent viologen-based EC mixture was developed following a procedure previously reported and patented by IK4-CIDETEC. ${ }^{14,15}$ The mixture comprising all the electroactive materials was optimized to fulfill the established ophthalmic requirements for optical contrast (at least 15-50\% transmittance), color (as colorless as possible when bleached), specific spectral absorption (blue should be filtered), transmittance stability in the colored state (for hours), and response times (faster than photochromatic lenses). A suitable EC mixture was prepared as follows: 1,1'-diethyl-4,4'-bipyridilium dibromide (0.77 wt\%), 1-butyl-3-methylimidazolium tetrafluoroborate $(43.87 \mathrm{wt} \%)$, and propylene carbonate $(54.84 \mathrm{wt} \%)$ were mixed for $1 \mathrm{~h}$ at room temperature. Next, ferrocene $(0.02 \mathrm{wt} \%)$ was added 
to the mixture, and then 5,10-dihydro-5,10-dimethylphenazine $(0.50 \mathrm{wt} \%)$. The resulting EC mixture was stirred for $15 \mathrm{~min}$.

Each EC device was composed of a sandwich of two polymeric lenses held by a plastic spacer to create a 150-micron thick cavity to host the electrochromic solution. Both lenses were coated with an antireflective multilayer coating on the external side and a transparent conductor coating (tin-doped indium oxide [ITO]) on the inner side. Coatings were applied using a physical vapor deposition machine with an ion-assisted deposition electron-beam gun using a Leybold CSS II coater with a Mark II ionbeam gun. ITO films $(130 \mathrm{~nm})$ were evaporated following a standard procedure, ${ }^{16}$ obtaining sheet resistances of $60-65 \Omega /$ sq. The device was filled by hand with the EC mixture under vacuum and sealed with photocurable adhesive. Wire-wrap cables were placed in the connection areas and attached with epoxy resin. Only the lens closest to the eye had optical power to correct near and distance ametropy with a multifocal lens, whereas the other was a standard plano powerless lens.

\subsubsection{Frame design}

Unigraphics software package was used to create a three-dimensional (3D) stl file of a frame that could host the electrochromic devices, the electronics to control them, and a pair of batteries (Fig. 1, top). Although many configurations were studied, for the purpose of simplicity the electronics were placed in the frame front and heel, while the batteries were placed in the temples. To simplify connections between the batteries and electronics, no hinges were
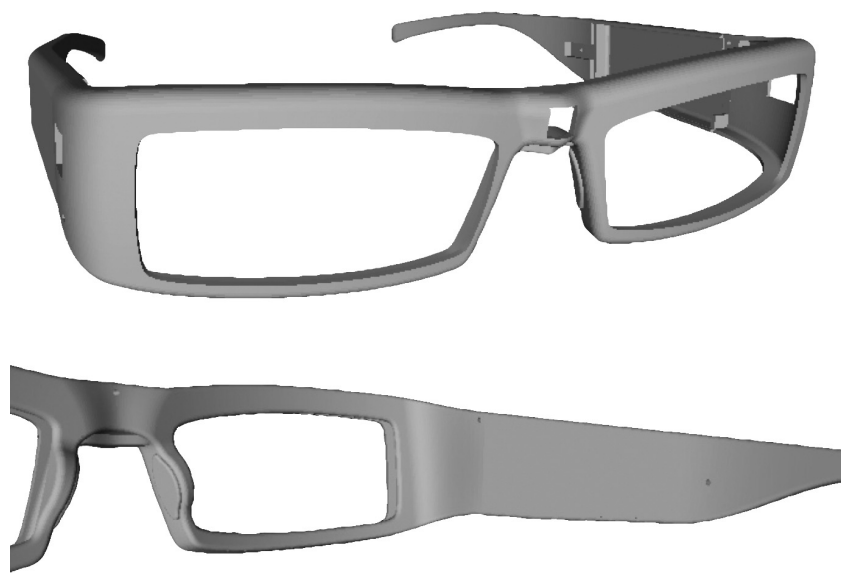

Fig. 1. 3D front and rear views of the front part of the frame. included. A back lid held by screws was designed to cover the electronics and batteries and fix the electrochromic devices (Fig. 1, bottom). Besides the functional spectacle requirements, the frame was also designed to be as aesthetically pleasing as possible while maintaining sufficient comfort for the widest possible range of users.

All the user-test frames were fabricated using laser-sintering technology on a standard polyamide material. After sintering, the frames were sand blasted and spray painted using standard acrylic paint for decoration.

\subsubsection{Driver electronics}

The main challenge of this system was the miniaturization of all the necessary electronics into the bridge, bar, heel, and temple of the glasses. Considering the signals involved, the number of connections crossing the bridge was minimized. The circuit was placed close to the EC devices along the bridge (ambient photodiode and its acquisition circuit), bar (wires for EC power and tracks for photodiode signal), and heel (control circuit) of the glasses, leaving a part to be connected to the temples, where two Li-Polymer batteries from Tempel ${ }^{\odot}$ were placed (Fig. 2). Microswitches selected the operation mode and the transmittance level. A micro-USB connector allowed the user charging batteries (with a devoted microchip controller) and also direct programming via $\mathrm{PC}$. The entire system was controlled by automatic PID and by usercontrolled algorithms using a Renesas Electronics ${ }^{\odot}$ 16-bit R8C microcontroller. As the frame of the glasses was curved, an all-flexible $4 \mathrm{~F}$ PCB was manufactured by WürthElectronik ${ }^{\odot}$ to support the circuit. Current consumption of approximately $12 \mathrm{~mA}$ was produced during circuit operation, with peaks of around $5 \mathrm{~mA}$ for each EC lens, and with a current of less than $1.5 \mathrm{~mA}$ for the lens to maintain coloration, resulting in several days of actual operation. Batteries can be charged from $0 \mathrm{~V}$ to $4.2 \mathrm{~V}$ in $2 \mathrm{~h}$ and $12 \mathrm{~min}$.

Two rear photodiodes, of the same model as the one of the bridge, were located behind the EC lenses. Its purpose was to control the EC lens coloring state. The microcontroller was able to compute the relationship between the central and rear photodiodes to obtain lens transmittance. Furthermore, it could also check the actual illumination reaching the eye during EC device bleaching or coloring. 


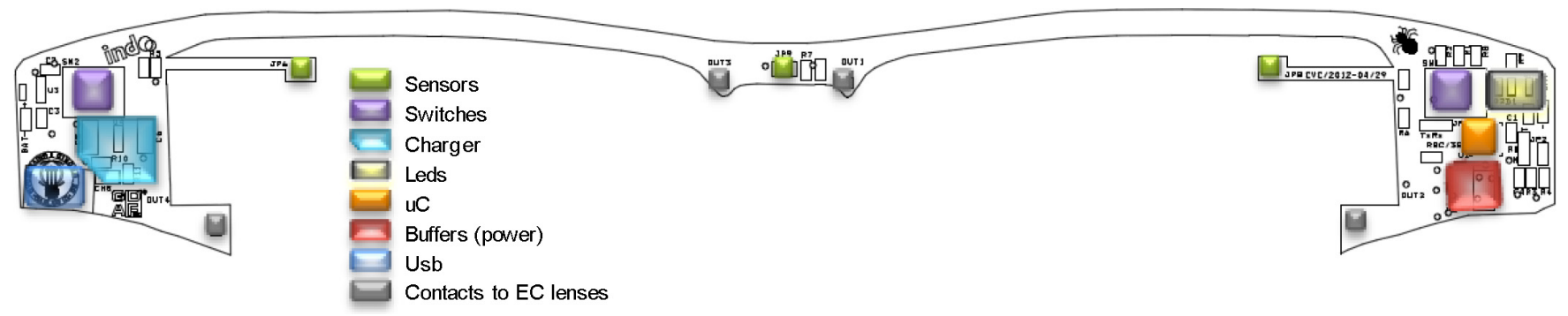

Fig. 2. Circuit layout. The placement of the different circuit parts is shown with a color LED to track the charge level (red, on whenever it should be recharged), the recharge state (yellow, on while recharging), and green to show the operation state (flickering the same number of times as the level number, when selecting one).

Although ready to work in RGB mode in the future, only green photodiodes were used in this study, due to its closer response to the human eye luminous intensity perception.

When using this device in automatic mode, programmed lighting that reaches the eye could be selected among 2000, 4000 or 6000 lux. Besides, the control system was programmed in the manual mode to regulate lens transmittance at four different levels: maximum clarity and $50 \%, 25 \%$, and $15 \%$ transmittance. Lenses were calibrated individually to achieve the programmed transmittance in the spectacle, as the refraction altered this measurement. Consequently, although the electronic system was able of measuring transmittance using the central and rear photodiodes, manual mode switching voltages were precalibrated for each spectacle. During the entire test, the system used the obtained voltage values without further correction.

\subsubsection{Clinical evaluation}

A glare measurement questionnaire was developed and validated previously to select the most appropriated patients to be included in the study (Annex 1).

The patient inclusion criteria were: uncomplicated cataract surgery with the same type of clear intraocular lens (IOL) in both eyes and at least a month post-op, score of 3 or higher on at least two of the nine questions on the ad hoc glare measurement questionnaire, binocular VA lower or equal to 0.1 LogMAR without distance refraction, and patients understanding and signing the consent form. The exclusion criteria were: other ocular pathology, neurological disorders (e.g., Alzheimer disease) or cognitive impairment, inability to read, and inability to meet the scheduled revisions.

A screening visit was performed to check inclusion/exclusion criteria, and to obtain the patient distance and near refraction to include in the electrochromic filter prototype (EFP).

The EFP was carried out and tested during four weeks (January and February) in a city in northern Spain, having both sunny and cloudy days. A second ad hoc questionnaire that was developed to measure the overall satisfaction when using the EFP was administered in the last visit (Annex 2). Its results were considered as the main outcome measures of the study.

Besides on the initial and final visits, distance best corrected VA (BCVA), contrast sensitivity (CS), and reading performance were measured. All those tests were performed in the same examination room, where standardized lighting conditions were ensured by blocking daylight.

BCVA was measured at a distance of $4 \mathrm{~m}$ with the ETDRS test (Lighthouse Low Vision Products, NY, USA) with the patient refraction in trial frame and the EFP in the different manual modes (Table 1). Measurements were done under different conditions, with an interval of at least 5 min between them. Background luminance was controlled by using three $4 \times 18 \mathrm{~W}$ daylight ceiling luminaries that allowed achieving approximately 200 lux at eye level. To provide glare, a $200 \mathrm{~W}$ halogen lamp was added, reaching approximately 4500 lux at eye level placed at $70 \mathrm{~cm}$ directed to the face, and about 1500 lux at $1.5 \mathrm{~m}$ from the face. To prevent fatigue effects, the test sequence was set from lower to higher brightness luminance conditions (Table 1).

To measure CS, Pelli-Robson test (Clement Clarke Intl/Haat-Streit, Harlow, UK) at $1 \mathrm{~m}$ was used, with the refraction in trial frame and with the EFP in off-mode. A $2 \times 15 \mathrm{~W}$ flexo fluorescent lamp was added to the background illumination mentioned before to obtain the test chart luminance recommended, $85 \mathrm{~cd} / \mathrm{m}^{2}$ measured using the Photometer Amprobe LM120 (Amprobe ${ }^{\circledR}$ Test Tools Europe, 
Table 1. Visual function outcomes (VA, CS, and RS) at the beginning and the end of the study, using standard correction and the electrochromic prototype filter, under different illumination conditions.

\begin{tabular}{|c|c|c|c|c|}
\hline & & Day 1 & Day 28 & $\mathrm{P}$ \\
\hline \multicolumn{5}{|l|}{ VA (ETDRS letters) } \\
\hline \multirow[t]{3}{*}{ Standard conditions } & $\mathrm{RX}$ & $86.4 \pm 3.65$ & $87.25 \pm 4.65$ & 0.5862 \\
\hline & $\mathrm{EC}$ & $88 \pm 3.67$ & $88.75 \pm 4.11$ & 0.7127 \\
\hline & $p$ & 0.3613 & 0.5862 & - \\
\hline \multirow[t]{3}{*}{ Illuminated } & $\mathrm{RX}$ & $87.4 \pm 2.3$ & $87.5 \pm 4.2$ & 0.8539 \\
\hline & EC & $87.8 \pm 3.11$ & $90 \pm 5.23$ & 0.0947 \\
\hline & $p$ & 0.7728 & 0.1814 & - \\
\hline \multirow[t]{3}{*}{$1000 \operatorname{lux}$} & $\mathrm{RX}$ & $88.2 \pm 3.7$ & $90.25 \pm 2.87$ & 0.1814 \\
\hline & $\mathrm{EC}-$ & $89 \pm 4.85$ & $88.75 \pm 6.13$ & 1 \\
\hline & $p$ & 0.5862 & 0.5839 & - \\
\hline \multirow[t]{6}{*}{4000 lux } & $\mathrm{RX}$ & $80 \pm 17.42$ & $87.75 \pm 1.89$ & 0.7893 \\
\hline & EC Man-Max & $86 \pm 4.3$ & $87 \pm 2.94$ & 0.5807 \\
\hline & EC Man-50 & $86.8 \pm 5.26$ & $86.5 \pm 8.1$ & 0.8501 \\
\hline & EC Man-25 & $86.4 \pm 5.13$ & $86.75 \pm 3.77$ & 1 \\
\hline & EC Man-12 & $87 \pm 4.85$ & $87.25 \pm 3.59$ & 0.8539 \\
\hline & $p$ & 0.8788 & 0.9985 & - \\
\hline \multicolumn{5}{|c|}{ CS (Pelli-Robson letters) } \\
\hline \multirow[t]{3}{*}{ Stantard conditions } & $\mathrm{RX}$ & $34.6 \pm 1.67$ & $33.75 \pm 2.87$ & 0.3711 \\
\hline & $\mathrm{EC}$ & $34.6 \pm 1.52$ & $34.75 \pm 1.89$ & 1 \\
\hline & $p$ & 1 & 1 & - \\
\hline \multirow[t]{3}{*}{ Glare } & $\mathrm{RX}$ & $34.2 \pm 3.03$ & $33.25 \pm 3.2$ & 1 \\
\hline & EC & $34.4 \pm 2.61$ & $34.5 \pm 2.38$ & 0.3458 \\
\hline & $p$ & 0.4237 & 1 & - \\
\hline \multirow[t]{3}{*}{ Reading (wpm) } & $\mathrm{RX}$ & $59.28 \pm 6.75$ & $62.58 \pm 11.93$ & 0.5839 \\
\hline & $\mathrm{EC}$ & $68.07 \pm 14.19$ & $69.33 \pm 8.42$ & 0.5839 \\
\hline & $p$ & 0.0591 & 0.5839 & - \\
\hline
\end{tabular}

Note: RX: User refractions in trial frame.

EC: Electrochromic prototype filter with user refraction.

EC: EC in mode off.

EC Man-Max: EC with manual transmittance at maximum transmittance.

EC Man-50: EC with manual transmittance at $50 \%$.

EC Man-25: EC with manual transmittance at $25 \%$.

EC Man-12: EC with manual transmittance at $25 \%$.

EC Man-12: EC with manual transmittance at $12 \%$.

Testing conditions:

- Standard conditions: Setting according tests manufacturer.

- Illuminated: Same as standard conditions with ambient light (200 lux at face level).

- 1000 lux: 1000 lux illumination at face.

- 4000 lux: 4000 lux illumination at face.

- Glare: Standard conditions adding halogen $50 \mathrm{~W}$ lamps at both sides of the test.

Germany). Next, glare was induced with two $50 \mathrm{~W}$ halogen lamps located on both sides of the PelliRobson test and directed toward the patient's eyes (Clement Clarke Intl/Haat-Streit, Harlow, UK).

Reading speed (RS) was measured with the near correction and with the progressive EFP in off-mode, under the ambient illumination cited above, and adding a $2 \times 15 \mathrm{~W}$ flexo fluorescent lamp directed toward the test (getting about 1000 lux on the test).
Reading performance was measured with the IReST reading test (Precision Vision, NY, USA), ${ }^{19}$ recording reading speed and errors committed.

After the initial visit, each customized EFP was delivered to the corresponding patient. Baseline, Visit 2 ( 7 days \pm 1 day), Visit 3 (14 days \pm 1 day), Visit 4 (21 days \pm 1 day), and final visit included an evaluation of glass behavior (measurement of possible transmittance changes at baseline and in manual 
modes: Man-Max, Man-50, Man-25, and Man-12). Filter transmittance under each condition was ascertained with the spectrometer USB2000+XR (Ocean Optics, Germany). To test the filter behavior, a switching time of 2 min was considered sufficient to achieve the lens transmittance fixed at each manual mode, so measurement with the spectrometer was performed at that time. The same voltages applied to achieve the transmittance levels obtained during the calibration were driven to check any spectral degradation in absorption after 2 min activation in each mode.

\subsubsection{Statistical analysis}

The distributions of quantitative variables are expressed as the mean, standard deviation, median, minimum, and maximum. The signed rank Wilcoxon test for paired data was used to compare the results between visits and between different conditions. For multiple comparisons of conditions lower than 4000 lux, an ANOVA nonparametric Friedman test was used. Qualitative variables were expressed as percentages with $95 \%$ confidence intervals.

\section{Results}

With respect to initial lens functionality, eight electrochromic filter prototypes were developed for clinical evaluation at the IOBA settings. One of them was inoperative and another two were considered unsuitable for the study as they did not provide transmittance enough. Consequently, only five pairs of lenses could be programmed to obtain the desired transmittances. Appearance and homogeneity of some glasses were inconstant, two of them having bubbles within the EC. As the size of the bubble was small, it was displaced upward and did not interfere with the primary gaze position, so the lenses could be tested. The bubbles seemed to remain stable in size during the study (Fig. 3).

After spectacle use over time, a transmittance increase was found in the manual modes 50, 25, and 12 (transmittances initially fixed for the green

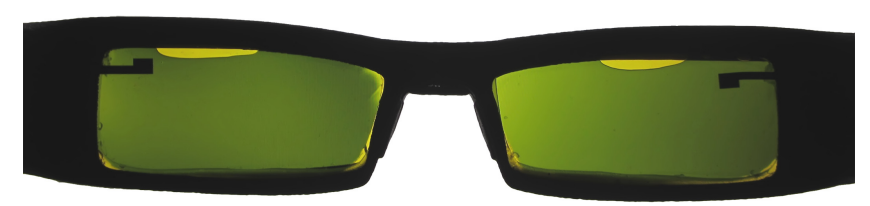

Fig. 3. Bubbles in one of the spectacles used. photodiode response, close to the $550 \mathrm{~nm}$ value; Fig. 4). Decrease of transmittance in the standby and Man-Max modes was also appreciated. The ratio of lower to higher wavelengths transmittance also changed, thus the lenses acquired a slightly different coloration.

\subsection{Clinical evaluation}

Five patients ( 1 women, 4 men) with a mean age of $73.2 \pm$ years (range: $72-85$ years) were included in the clinical evaluation of the electrochromic glasses. All five patients were pseudophakic, and had no posterior capsule opacity, except one who received YAG capsulotomy in both eyes. The average binocular BCVA values were between 86 and 90 ETDRS letters (20/ 25+1-20/16 in Snellen scale) (Table 1). No statistically significant differences between first and last visits were found in any lighting condition used. No significant differences in BCVA between measurements with normal glasses and the prototype were found at any visit. Likewise, no differences were found either in BCVA measured under monocular or binocular conditions $(p \geq 0.10)$. Concerning CS, no statistically significant differences were found between first and last visits nor between the different lighting conditions tested for both trial frame corrections or with the prototype ( $p \geq 0.10$, Table 1$)$.

There were no significant differences between initial and final visits in the time spent to read a paragraph and in the number of errors committed both with and without the prototype (Table 1). When comparing the two devices, there were also no significant differences. However, the time spent with the electrochromic prototype filter was higher and near the limit of statistical significance when compared to the time spent with their glasses at the first visit $(p=0.06)$.

\subsection{Satisfaction questionnaire results}

Before the study, only one person used distance glasses and two near vision glasses. Two people used sunglasses, both with neutral lenses, one of them being tinted gray and the other brown gradient. Once patients had the prototype, four of them used it daily, and one used for 2-3 days/week. The main activity for which the prototype was used was walking $(n=4)$. Figure 5 shows the time in minutes that patients spent in different activities wearing the prototype tested. 


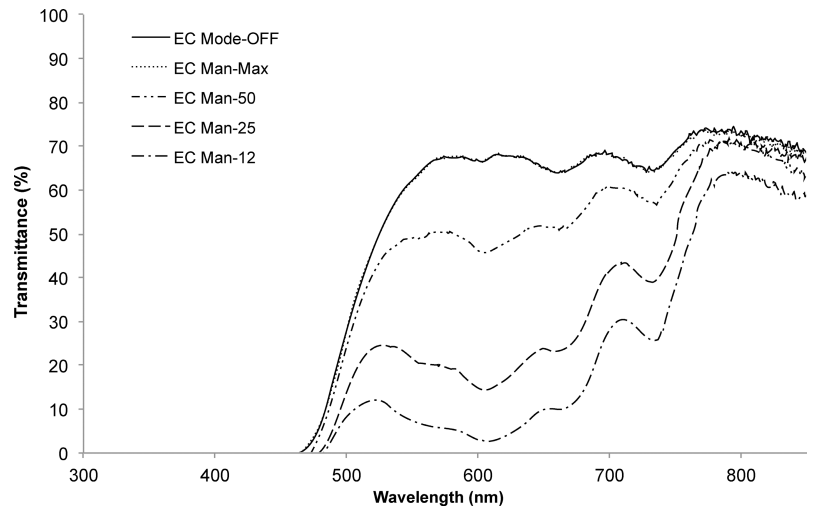

(a)

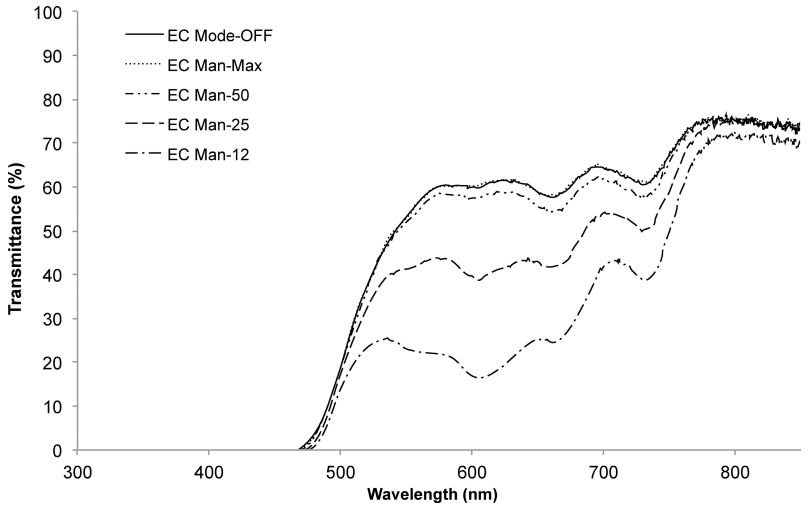

(b)

Fig. 4. Example of transmittance change of lenses used in the study: (a) Lens 9 (refraction $=+0.50 \mathrm{sph}-0.75 \mathrm{cyl} \times 120^{\circ}$ ) after adjusting the transmittance to the refraction, using the assistance software; (b) Transmittance change after 2 min activation in each mode and after one month of use.

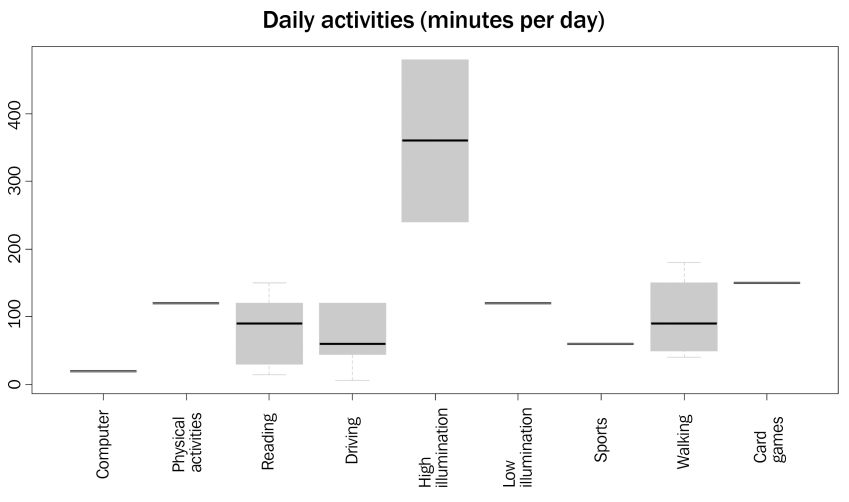

Fig. 5. Time spent developing different daily activities by patients wearing the prototypes evaluated in the study.

The user ratings showed various aspects of electrochromic prototype filter during use. The first value refers to the users' general opinion about the prototype filter. They found the frame comfortable and the manual mode useful, and thought that the battery indicator, the way of charging the battery, and the automatic mode were impractical (Fig. 6). Lens performance was assessed as intermediate in all features (speed of change, level of darkness/ lightness and color aesthetics), as shown in Figs. 7-9. Only one individual found that the dimming level was insufficient. Another individual did not know if the self-dimming was the same as the first day. The remaining variables (sufficient level of transparency, same speed than the first day, and similar behavior in both lenses) were unchanged and equal to "Yes" for the five users.

Patients were asked about influence of the EFP in color and contrast perception. Only one out of

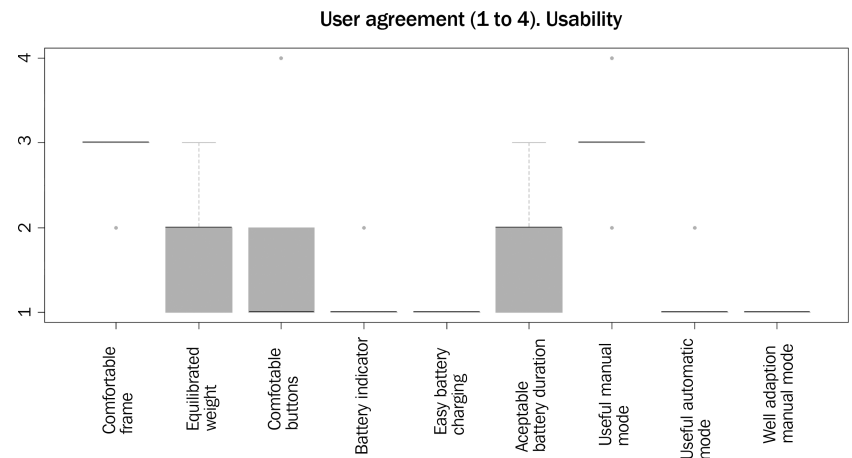

Fig. 6. Usability of the electrochromic prototype filter. Responses rated the degree of agreement with the statements rated by the user on a scale of $1-4$ ( $1=$ strongly agree, $2=$ somewhat agree, $3=$ somewhat disagree, and $4=$ strongly disagree).

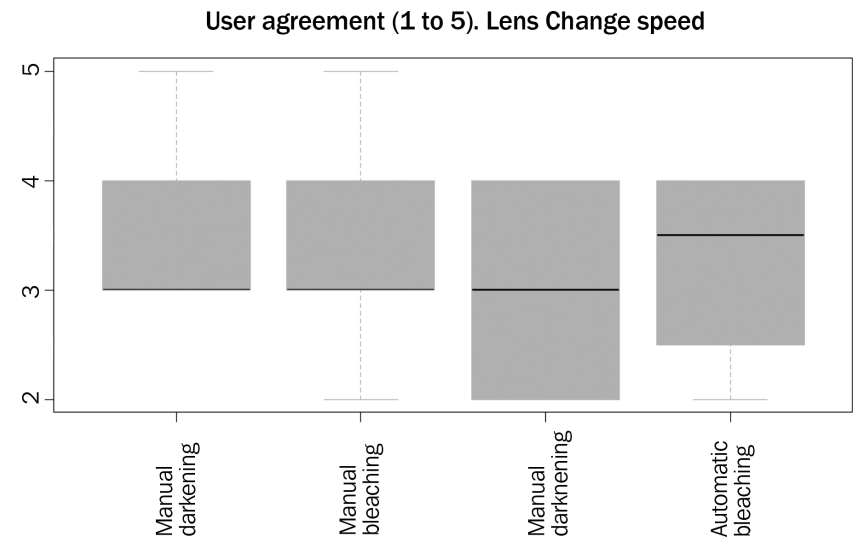

Fig. 7. Users' assessments of the lens change speed. Likert scale $1-5$ ( $1=$ very fast, $2=$ fast, $3=$ intermediate, $4=$ slow, and $5=$ very slow). 
User agreement (1 to 5). Darkness/Lightness maxumim level

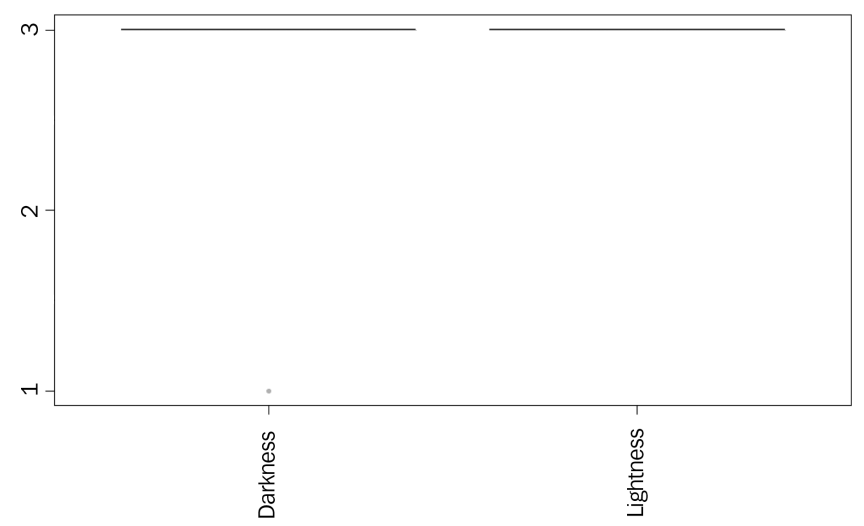

Fig. 8. Users' assessments of the maximum level of lens darkness/lightness. Likert scale $1-5$ ( $1=$ very clear, $2=$ clear, $3=$ normal, $4=$ dark, and $5=$ very dark).

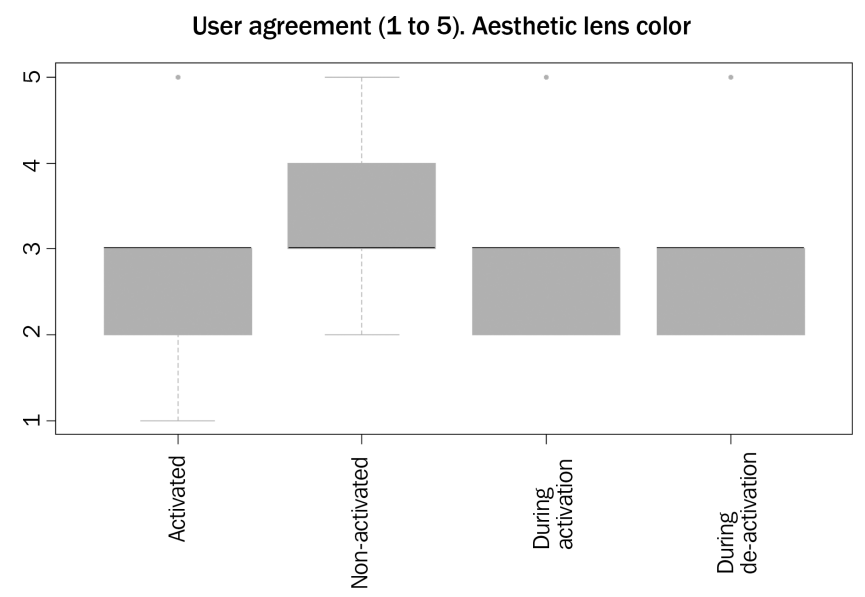

Fig. 9. Users' assessments of the lens color aesthetics. Likert scale $1-5$ ( $1=$ cosmetic, $2=$ relatively aesthetic, $3=$ normal, $4=$ relatively unsightly, and $5=$ very ugly).

five found that color changed, but he was more comfortable with it because contrast improved.

No patients reported variable discomfort when passing tunnels. Likewise, all patients answered "No" to the following statements: "not sufficiently clear indoors", "not dark in the car", "put on and remove discomfort", and "other problems". Regarding "excessive time to get dark", "excessive time to clarify", and "not dark enough under extreme lighting", only one user reported having problems.

\section{Discussion}

From a technical perspective, we succeeded in achieving five properly working prototypes, despite of the hand manufacturing of the two crystals containing the EC. This manufacturing can be considered as a limiting factor for the correct working of the prototypes, as some lost the EC. and others had bubbles affecting the visual axis. Throughout the study, crystal transmittance varied under the same voltage conditions (Fig. 4) which is important to assess the prototype durability. This change was noted by patients, reaching all prototypes a satisfactory degree of darkness throughout the entire study. From our perspective, the prototype still has some room for improvement as it has shown a satisfactory performance in our study.

Our results also show that the activation and specially bleaching times of the EC devices described are competitive with respect to photochromatic lenses. The electronic circuit, including battery autonomy, weight, and size considerations, shows the feasibility of inserting everything into the spectacles, controlling the EC lenses with a green photodiode response, and using a PID algorithm to maintain user-desired coloration. A custom control is also possible by programming the circuit microcontroller, ad hoc for each user, via a micro-USB port also used for battery recharge. The users found response independence to temperature conditions very useful: photochromatic are very sensitive to temperature, but EC redox reaction is produced inside the lenses with a negligible dependence on it. Although the prototypes were set up for both manual and automatic modes to achieve dimming, patients preferred the manual one. This duality offered by the prototype should be evaluated further, because if the automatic mode does not work well, it should be either improved or removed to simplify the device. As automatic mode works to maintain a certain illuminance value at the eye, maybe the luxes levels programmed were not sufficient for patient comfort under sunny conditions, thus leading to his/her switching to manual mode to darken the devices. With the same values, during indoor operation, automatic mode did not switch the devices, leading also to discomfort in the user accommodation.

Concerning the clinical outcomes, our study confirms that VA, CS, and reading ability did not decrease with the prototype use. Therefore, the quality of vision provided by the prototype was good and comparable to that provided by conventional correction on a trial frame. Likewise, the prototype provided a successful correction of near vision in the pseudophakic eyes evaluated. This is a good outcome considering that conventional glasses 
in pseudophakic eyes may have a limited impact on vision. ${ }^{17}$ Our outcomes suggest that this type of filters may be an integrated solution for solar radiation protection and presbyopia. To our knowledge, this is the first clinical study evaluating a filter with these specific properties. Future studies with larger samples sizes must confirm these promising results obtained in our pilot study.

The satisfaction questionnaire showed that all patients used the prototype, most of them daily. This high level of usage may indicate that the prototypes were not only comfortable but also useful. Patients used them mainly for walking, which may mean that the prototype was preferred for outdoor activities. Patients were generally satisfied and found that this solution offered advantages compared with the previous situation where they wore several glasses. Future comparative studies must confirm the potential superiority of the prototype developed over photochromic filters which have shown already benefits in terms of vision-related quality of life (VRQOL). ${ }^{18}$ Finally, patients found an additional subjective advantage in improved contrast, although we found no differences in contrast sensitivity measured with or without the prototype.

In conclusion, our pilot study shows that electrochromic-technology filtering glasses may be acceptable as a single device that works properly for outdoor/indoor use and for distance-near vision, although they still need improvements. Future studies in larger sample sizes should confirm the clinical outcomes with these filters in the long-term as well as their superiority over other type of filtering lenses.

\section{Acknowledgments}

This work was supported by the Project "Customized Eye Care", CEYEC, CEN-20091021 from the Spanish "Centro para el Desarrollo Tecnológico Industrial" (CDTI) in the fifth call of the CENIT-E program, by Comunidad Autónoma de Madrid under the FACTOTEM2 project, S2009/ESP-1781 and also by the R\&D Program SINFOTON S2013/MIT-2790. We thank the support from the IOBA's statistical Unit, particularly to Itziar Fernandez.

\section{References}

1. M. Ao et al., "Color discrimination by patients with different types of light-filtering intraocular lenses," $J$. Cataract Refractive Surg. 36, 389-395 (2010).
2. J. Schwiegerling, "Recent developments in pseudophakic dysphotopsia," Curr. Opin. Ophthalmol. 17, 27-30 (2006).

3. F. Eperjesi, L. E. Agelis, "Effects of yellow filters on visual acuity, contrast sensitivity and reading under conditions of forward light scatter," Graefe's Arch. Clin. Exp. Ophthalmol. 249, 709-714 (2011).

4. H. Yang, N. A. Afshari, "The yellow intraocular lens and the natural ageing lens," Curr. Opin. Ophthalmol. 25, 40-43 (2014).

5. I. Schmack, M. Schimpf, A. Stolzenberg, I. ConradHengerer, F. H. Hengerer, H. B. Dick, "Visual quality assessment in patients with orange-tinted blue light-filtering and clear ultraviolet light-filtering intraocular lenses," J. Cataract Refractive Surg. 38, 823-832 (2012).

6. H. Wang, J. Wang, W. Fan, W. Wang, "Comparison of photochromic, yellow, and clear intraocular lenses in human eyes under photopic and mesopic lighting conditions," J. Cataract Refractive Surg. 36, 20802086 (2010).

7. R. W. Nowakowski, Primary Low Vision Care, Appleton \& Lange, Norwalk, p. 374 (1994).

8. M. Born, E. Wolf, Principles of Optics: Electromagnetic Theory of Propagation Interference and Diffraction of Light, 7th Edition, Cambridge University Press Cambridge, p. 952 (2006).

9. C. M. Lampert, "Chromogenic smart materials," Mater. Today 7, 28-35 (2004).

10. D. Takahashi, PixelOptics shows off electronicfocusing eyeglasses (video), http://venturebeat.com/ 2012/01/13/pixeloptics-shows-off-electronic-focusing-eyeglasses-video/, Accessed on 31 August 2015.

11. C. Ma, M. Taya, C. Xu, "Smart sunglasses with tunable shade," SPIE Proc. 6927, 69271J (2008), doi: $10.1117 / 12.776596$.

12. C. Ma, C. Xu, "Smart goggles based on all-plastic electrochromic devices," SPIE Proc. 7493, 74931C (2009), doi: 10.1117/12.844576.

13. P. M. S. Monk, R. J. Mortimer, D. R. Rosseinsky, Electrochromism and Electrochromic Devices, Cambridge University Press, Cambridge, p. 483 (2007).

14. C. P. Gonzalo, R. M. Garcia, M. S. Telleria, J. A. P. Alonso, H. J. G. Telleria, Viologen-based electrochromic compositions which can be formulated and applied at room temperature, Google Patents (2011).

15. C. Pozo-Gonzalo, M. Salsamendi, A. Viñuales, J. A. Pomposo, H. J. Grande, "Highly transparent electrochromic plastic device that changes to purple and to blue by increasing the potential," Sol. Energy Mater. Sol. Cells 93, 2093-2097 (2009).

16. S. P. Hurley, Liquid crystal displays for pixelated glare shielding eyewear, Ph.D. Thesis, Kent State University (2010), https://etd.ohiolink.edu/rws_etd/ document/get/kent1279550994/inline, Accessed on 22 February 2016. 
17. J. Maki, S. Kusakul, K. Morley, T. Sanguansak, J. Seddon L. Hartung, M. Morley, "The effect of glasses on visual function following cataract surgery in a cataract camp," Br. J. Ophthalmol. 92, 883-887 (2008).

18. S. Stenson, K. Scherick, C. J. Baldy, K. A. Copeland, J. Solomon, C. Bratteig, "Evaluation of vision- related quality of life of patients wearing photochromic lenses," CLAO J 28, 128-135 (2002).

19. Trauzettel-Klosinski S1, Dietz K; IReST Study Group, "Standardized assessment of reading performance: The New International Reading Speed Texts IReST." Invest Ophthalmol Vis Sci. 53, 54525461 (2012). 